\title{
INFLUÊNCIA DA INVASÃO TUMORAL DA LINHA DE ANASTOMOSE NA SOBREVIVÊNCIA DE PACIENTES COM CÂNCER DE COTO GÁSTRICO
}

\author{
The influence of tumor invasion in anastomotic line on survival of patient with \\ gastric stump cancer
}

\author{
Ana Lúcia Granja Scarabel Nogueira CARRASCO, Cláudio José Caldas BRESCIANI, \\ Rodrigo Oliva PEREZ, Bruno ZILBERSTEIN, Ivan CECCONELLO
}

ABCDDV/592

Carrasco ANGSN, Bresciani CJC, Perez RO, Zilbertein B, Cecconello I. Influência da invasão tumoral da linha de anastomose na sobrevivência de pacientes com câncer de coto gástrico. ABCD Arq Bras Cir Dig. 2008;21(2):55-60

RESUMO - Racional - O câncer do coto gástrico desenvolve- se no remanescente gástrico de gastrectomia realizada há pelo menos 5 anos por doença benigna e os sítios mais comuns de acometimento são próximo à anastomose e na pequena curvatura. Considera-se que o coto gástrico é estado pré-canceroso. Objetivos - Identificar o padrão de disseminação de linfonodos acometidos, quantificar a invasão tumoral da linha de anastomose e correlacionar: a invasão da linha de anastomose com o comprometimento linfonodal e mesenterial, o acometimento linfonodal com sobrevivência e o acometimento da linha de anastomose com sobrevivência. Métodos - Estudo retrospectivo com revisão de prontuários, peças cirúrgicas e exames anátomo-patológicos de 113 pacientes com diagnóstico de câncer de coto gástrico definido como adenocarcinoma desenvolvido no remanescente gástrico de gastrectomia realizada há pelo menos cinco anos por doença benigna. Resultados - A disseminação linfonodal não se mostrou específica; $75 \%$ dos pacientes apresentaram invasão tumoral da linha de anastomose; em $66,7 \%$ dos casos ocorreu invasão da linha anastomótica e linfonodal concomitantes; menos de $10 \%$ dos casos exibiam invasão mesenterial; houve óbito em $86,5 \%$ dos casos com invasão linfonodal e $64,7 \%$ com invasão da linha de anastomose e em 100\% com invasão mesenterial. Conclusões - 1) O câncer de coto gástrico não tem padrão de disseminação linfonodal específico; 2) a linha de anastomose sofre freqüente invasão tumoral;3) apesar de freqüente a invasão da linha anastomótica, não apresenta correlação estatística significante com o comprometimento linfonodal regional ou mesenterial; 4) a presença de invasão linfonodal implica em sobrevida menor, em especial a de linfonodos do mesentério; 5) a presença de acometimento neoplásico da linha anastomótica não se correlaciona com pior resultado de sobrevivência.

DESCRITORES: Coto gástrico. Anastomose cirúrgica. Sobrevivência, cirurgia. Câncer gástrico.

\section{INTRODUÇÃO}

O câncer do coto gástrico é definido como adenocarcinoma desenvolvido no remanescente gástrico de gastrectomia realizada há pelo menos cinco anos por doença benigna. A doença descrita pela primeira vez em 1922 por Balfour e Tofgaard ${ }^{30}$, aumenta de incidência após 20 anos de gastrectomia33 e reconstrução gástrica, principalmente pela técnica a Billroth $\mathrm{II}^{2,4,13,17}$.

Vários autores acreditam que a gastrectomia seja um estado pré-canceroso $6,8,10,19,20,25,26,29,34$.

Os sítios no estômago mais comuns de acometimento pelo câncer gástrico são a região próxima à anastomose ${ }^{6,12,32}$ e a pequena curvatura ${ }^{22,23}$. Ele representa de 1 a $9 \%$ dos casos de câncer do estômago ${ }^{7,21}$ com prevalência de 0,8

Trabalho realizado na Disciplina de Cirurgia do Aparelho Digestivo do Departamento de Gastroenterologia da Faculdade de Medicina da Universidade de São Paulo, Serviço de Cirurgia do Estômago e Intestino Delgado da Divisão de Clínica Cirúrgica II do Hospital das Clínicas da Faculdade de Medicina Universidade de São Paulo e Programa de Pós-Graduação de Cirurgia do Aparelho Digestivo, São Paulo, SP, Brasil.

Endereço para correspondência: Ana Lúcia Granja Scarabel Nogueira Carrasco. E-mail: ana.carrasco@globo.com.
$8,9 \%$ em indivíduos previamente submetidos à ressecção gástrica parcial por lesão benigna ${ }^{33}$.

A principal teoria de carcinogênese é a que supõe que o refluxo enterogástrico provocaria alteração da mucosa do coto gástrico e evoluiria com processo crônico inflamatório até a gastrite atrófica, decorrendo daí o aparecimento da metaplasia intestinal e posteriormente a displasia ${ }^{5,6,7,11,14,15,27,33}$. Outros mecanismos potenciais para carcinogênese são hipo ou acloridria; resposta à proliferação aumentada devido à gastrite crônica; efeito da regulação hormonal e hipergastrinemia após vagotomia e presença de sutura cirúrgica ${ }^{24}$.

$\mathrm{O}$ estadiamento do câncer de coto gástrico segue as regras do câncer gástrico, uma vez que, não há até o momento, regras específicas para câncer de coto gástrico. No momento do diagnóstico a presença de linfonodos acometidos pode ser alta, o que sombreia a perspectiva de sobrevivência dos pacientes. E a gastrectomia prévia pode induzir uma série de alterações anatômicas com as aderências cicatriciais que podem eventualmente comu- 
nicar áreas distintas de drenagem linfática, influenciando os resultados do tratamento cirúrgico dessa doença em relação ao adenocarcinoma do estômago nunca operado ${ }^{9}$. Por outro lado, alguns autores julgam que o prognóstico do câncer de coto gástrico é melhor que do câncer gástrico, possivelmente porque a fibrose e a interrupção de algumas vias de disseminação linfática dificultariam a difusão da doença ${ }^{3,22}$.

A localização do tumor no estômago é fator prognóstico, sendo os do terço superior gástrico com pior evolução pela sua tendência à invasão esofágica e metástases linfonodais ${ }^{1,16,18}$. Por outro lado é possível que tumores originados próximo à linha de anastomose, mas que não a tenha invadido tenham melhor prognóstico em relação aos que tenham invadido o intestino delgado e disseminam metástase para linfonodos mesentéricos e diminuem o tempo de sobrevivência (cerca de 13,2 meses) ${ }^{31}$. Entretanto a fibrose da linha anastomótica pode apresentar barreira à difusão do tumor em direção ao intestino delgado. Por outro lado a invasão freqüente de órgãos adjacentes, a invasão do jejuno e as novas rotas de disseminação linfática podem explicar o prognóstico sombrio desse câncer quando em estádio avançado ${ }^{3}$. Estudos confirmam rotas de disseminação linfonodal em direção aos linfonodos mediastinais, ao mesentério ${ }^{28,35}$. Este fato sugere que a técnica de linfadenectomia no câncer de coto gástrico deva ser diferente e atender a estas peculiaridades da difusão linfonodal.

Os objetivos desse trabalho foram: identificar o padrão de disseminação de linfonodos acometidos, quantificar a invasão tumoral da linha de anastomose, correlacionar a invasão da linha de anastomose com o comprometimento linfonodal e mesenterial, correlacionar o acometimento linfonodal com sobrevivência e correlacionar o acometimento da linha de anastomose com sobrevivência.

\section{MÉTODOS}

Este estudo é retrospectivo com revisão de 113 prontuários, peças cirúrgicas e exame anátomo-patológicos. Foram elegíveis para inclusão pacientes com diagnóstico de câncer de coto gástrico definido como adenocarcinoma desenvolvido no remanescente gástrico de gastrectomia realizada há pelo menos cinco anos por doença benigna $\mathrm{e}$ terem sido submetidos à gastrectomia com linfadenectomia para permitir o conhecimento da distribuição do acometimento linfonodal.

Foram preenchidos protocolos de identificação do paciente e onde foram anotados dados pertinentes seja da história clínica, exames físico, pré-operatórios e histológicos, resultados de endoscopia, relatório cirúrgico, exame anátomo-patológico final, necessidade de novos procedimentos diagnósticos e terapêuticos e seguimento tardio, presença de queixas digestivas, recidiva tumoral, tempo livre de doença e tempo de sobrevivência. $\mathrm{O}$ estadiamento realizado seguiu o que foi proposto pela União Internacional Contra Câncer (1997) e obedeceu à classificação TNM e os linfonodos regionais foram designados segundo as regras do Japanese Gastric Cancer Association (1998).
Os parâmetros coletados na análise foram: presença ou não de invasão da linha de anastomose, distribuição do acometimento linfonodal, ocorrência de metástases em linfonodos do mesentério e sobrevida estudada conforme a presença ou não de invasão da linha de anastomose.

Foram formados dois grupos de estudo: um grupo composto de doentes que apresentaram invasão da linha anastomótica pelo tumor e outro constituído de doentes com tumor sem invasão da alça jejunal. O padrão de acometimento linfonodal foi quantificado em ambos os grupos assim como foi determinada a distribuição conforme $o$ estadiamento obtido nos dois grupos de estudo.

Realizou-se estudo comparativo do acometimento linfonodal entre os grupos constituídos, correlação entre a invasão da linha de anastomose com o comprometimento linfonodal-mesentérico, correlação entre o acometimento linfonodal com a sobrevivência assim como foram comparadas as curvas de sobrevivência obtidas dos dois grupos.

\section{RESULTADOS}

Foram analisados 113 prontuários, entretanto 41 foram excluídos por não se ter conseguido recuperar parâmetros definidos para análise. Portanto, 72 casos integraram o estudo.

A idade variou entre 36 e 89 anos, a mediana foi de 65,4 anos. Eram do sexo masculino 58 pacientes $(80,6 \%)$ e 14 do feminino (19,4\%). Em relação à raça, 64 eram da branca $(88,8 \%), 5$ da negra $(6,9 \%)$ e 3 da amarela $(4,2 \%)$. O acometimento linfonodal ocorreu em 42 pacientes (58,3\%), em $23(31,9 \%)$ não havia linfonodos acometidos e sete $(9,7 \%)$ não tiveram comprovação de invasão linfonodal, como mostra a Tabela 1.

TABELA 1 - Distribuição dos casos quanto à presença ou não de comprometimento linfonodal.

\begin{tabular}{lccc}
\hline $\begin{array}{l}\text { INVASÃO } \\
\text { LINFONODAL }\end{array}$ & No. CASOS & PERCENTUAL & $\begin{array}{c}\text { PERCENTUAL } \\
\text { VÁLIDO }\end{array}$ \\
\hline Não & 23 & $31,9 \%$ & $35,4 \%$ \\
Sim & 42 & $58,3 \%$ & $64,6 \%$ \\
Total & 65 & $90,3 \%$ & $100 \%$ \\
Não disponível & 7 & $9,7 \%$ & - \\
\hline TOTAL & $\mathbf{7 2}$ & $\mathbf{1 0 0} \%$ & - \\
\hline
\end{tabular}

O padrão de disseminação linfonodal, seguindo as cadeias linfonodais regionais, encontra-se na Tabela 2.

A distribuição dos pacientes quanto à invasão tumoral da linha de anastomose está representada na Tabela 3.

Observou-se 54 casos com invasão (75\%), 15 sem invasão da linha anastomótica $(20,8 \%)$ e três casos sem esse dado preciso (4,2\%). A correlação entre invasão neoplásica da linha anastomótica e comprometimento linfonodal está representada na Tabela 4.

Nota-se que 32 pacientes $(66,7 \%)$ apresentavam comprometimento neoplásico concomitante da linha de anastomose e linfonodal. Comparado com o grupo que não teve invasão linfonodal concomitante com invasão tumoral 
TABELA 2 - Distribuição do padrão de disseminação do acometimento de linfonodos regionais.

\begin{tabular}{lc}
\hline CADEIAS LINFONODAIS & NO CASOS \\
\hline 1. Cárdica direita & 1 \\
2. Cárdica esquerda & 1 \\
3. Pequena curvatura & 9 \\
4. Grande curvatura & 5 \\
5. Suprapilórica & 1 \\
6. Infrapilórica & - \\
7. Artéria gástrica esquerda & 1 \\
8. Artéria hepática comum & 2 \\
9. Tronco celíaco & 7 \\
10. Hilo esplênico & 4 \\
11. Artéria esplênica & 1 \\
12. Ligamento hepatoduodenal & - \\
13. Retropancreática & - \\
14. Raiz do mesentério & 7 \\
15. Artéria cólica média & - \\
16. Para-aórtica & 2 \\
17. Pancreática anterior & 1 \\
18. Pancreática inferior & - \\
19. Infra-diafragmática & - \\
20. Hiato esofágico & - \\
110. Paraesofágica & - \\
111. Supradiafragmática & 2 \\
112. Mediastinal posterior & - \\
perigástrica/ outras & $11 / 10$ \\
\hline &
\end{tabular}

TABELA 3 - Quantificação da invasão tumoral da linha de anastomose em número absoluto de casos

\begin{tabular}{lccc}
\hline $\begin{array}{l}\text { INVASÃO TUMOR- } \\
\text { ALDALINHAANAS- } \\
\text { TOMÓTICA }\end{array}$ & No. CASOS & PERCENTUAL & $\begin{array}{c}\text { PERCENTUAL } \\
\text { VÁLIDO }\end{array}$ \\
\hline Sim & 15 & $20,8 \%$ & $21,7 \%$ \\
Não & 54 & $75,0 \%$ & $78,3 \%$ \\
Total & 69 & $95,8 \%$ & $100 \%$ \\
Não disponível & 3 & $4,2 \%$ & - \\
\hline TOTAL & $\mathbf{7 2}$ & $\mathbf{1 0 0} \%$ & - \\
\hline
\end{tabular}

TABELA 4 - Correlação da invasão tumoral da linha de anastomose com invasão linfonodal

\begin{tabular}{cccccc}
\hline & \multicolumn{5}{c}{ COMPROMETIMENTO LINFONODAL } \\
& & Não & Sim & Total \\
\hline & \multirow{2}{*}{ Não } & No. casos & 6 & 8 & 14 \\
& & $42,9 \%$ & $57,1 \%$ & $100 \%$ \\
& & Total & $9,7 \%$ & $12,9 \%$ & $22,6 \%$ \\
\cline { 2 - 6 } INVASÃO TUMORAL & No casos & 16 & 32 & 48 \\
DA LINHA & \multirow{2}{*}{ Sim } & $\%$ & $33,3 \%$ & $66,7 \%$ & $100 \%$ \\
ANASTOMÓTICA & & Total & $25,8 \%$ & $51,6 \%$ & $77,4 \%$ \\
\cline { 2 - 6 } & \multirow{2}{*}{ Total } & No. casos & 22 & 40 & 62 \\
& & $35,5 \%$ & $64,5 \%$ & $100 \%$ \\
\hline
\end{tabular}

da linha anastomótica $(57,1 \%)$, a diferença estatística não foi significante $(P<0,512)$. A correlação entre invasão neoplásica da linha de anastomose e comprometimento linfonodal mesenterial está representada na Tabela 5 .

A presença conjunta da invasão tumoral da linha de anastomose com o comprometimento linfonodal mesenterial esteve presente somente em 4 casos $(9,3 \%)$. Comparando com o grupo sem invasão linfonodal mesenterial a diferença estatística não foi significante $(P<0,149)$.
TABELA 5 - Correlação entre a invasão tumoral da linha de anastomose e comprometimento linfonodal mesenterial

\begin{tabular}{|c|c|c|c|c|c|}
\hline & & \multicolumn{4}{|c|}{$\begin{array}{c}\text { COMPROMETIMENTO } \\
\text { LINFONODAL MESENTERIAL }\end{array}$} \\
\hline & & & Não & Sim & Total \\
\hline \multirow{8}{*}{$\begin{array}{c}\text { INVASÃO TUMORAL } \\
\text { DA LINHA } \\
\text { ANASTOMÓTICA }\end{array}$} & \multirow{3}{*}{ Não } & No. casos & 9 & 3 & 12 \\
\hline & & $\%$ & $75 \%$ & $25 \%$ & $100 \%$ \\
\hline & & Total & $16,4 \%$ & $5,5 \%$ & $21,8 \%$ \\
\hline & \multirow{3}{*}{ Sim } & No casos & 39 & 4 & 43 \\
\hline & & $\%$ & $90,7 \%$ & $9,3 \%$ & $100 \%$ \\
\hline & & Total & $70,9 \%$ & $7,3 \%$ & $78,2 \%$ \\
\hline & \multirow{2}{*}{ Total } & No. casos & 48 & 7 & 55 \\
\hline & & $\%$ & 87,3 & $12,7 \%$ & $100 \%$ \\
\hline
\end{tabular}

Dos 72 pacientes estudados, 37 (61,7\%) apresentaram comprometimento linfonodal e desses $32(86,5 \%)$, morreram pela progressão da doença e $18(30,0 \%)$ não apresentaram comprometimento linfonodal ou estavam vivos.

Observou-se diferença estatística significante $(P<$ $0,001)$ em relação aos pacientes com comprometimento linfonodal e que morreram e aqueles pacientes sem comprometimento linfonodal e que sobreviveram (Tabela 6).

TABELA 6 - Correlação entre comprometimento linfonodal e óbito pela doença

\begin{tabular}{|c|c|c|c|c|c|}
\hline & & \multicolumn{4}{|c|}{ ÓBITO } \\
\hline & & & Não & Sim & Total \\
\hline \multirow{8}{*}{$\begin{array}{l}\text { COMPROMETIMENTO } \\
\text { LINFONODAL }\end{array}$} & \multirow{3}{*}{ Não } & No. casos & 18 & 5 & 23 \\
\hline & & $\%$ & $78,3 \%$ & $21,7 \%$ & $100 \%$ \\
\hline & & Total & $30,0 \%$ & $8,3 \%$ & $38,3 \%$ \\
\hline & \multirow{3}{*}{ Sim } & No casos & 5 & 32 & 37 \\
\hline & & $\%$ & $13,5 \%$ & $86,5 \%$ & $100 \%$ \\
\hline & & Total & $8,3 \%$ & $53,3 \%$ & $61,7 \%$ \\
\hline & \multirow{2}{*}{ Total } & No. casos & 23 & 37 & 60 \\
\hline & & $\%$ & $38,3 \%$ & $61,7 \%$ & $100 \%$ \\
\hline
\end{tabular}

A média de sobrevivência dos pacientes com comprometimento linfonodal foi de 44,3 meses e em 60 meses havia $18,1 \%$ dos pacientes vivos. Sem comprometimento linfonodal a média de sobrevivência foi 150,2 meses. Aos 60 meses a sobrevivência era de 52,6\%.

Dos 51 pacientes com invasão tumoral da linha de anastomose, $33(64,7 \%)$ foram a óbito pela doença e 18 sobreviveram (35,3\%). Quando se compara o grupo de pacientes que morreu com e sem invasão tumoral da linha de anastomose, não há diferença estatística significante $(P<0,889)$ (Tabela 7).

TABELA 7 - Correlação entre a invasão tumoral da linha anastomótica e óbito pela doença

\begin{tabular}{cccccc}
\hline & & \multicolumn{4}{c}{ ÓBITO } \\
& & & Não & Sim & Total \\
\hline & & No. casos & 5 & 10 & 15 \\
& Não & $\%$ & $33,3 \%$ & $66,7 \%$ & $100 \%$ \\
COMPROMETIMENTO & & Total & $7,6 \%$ & $15,2 \%$ & $22,7 \%$ \\
\cline { 2 - 6 } LINFONODAL & \multirow{2}{*}{ Sim casos } & 18 & 33 & 51 \\
& & $\%$ & $35,3 \%$ & $64,7 \%$ & $100 \%$ \\
\cline { 2 - 6 } & \multirow{2}{*}{ Total } & No. casos & $27,3 \%$ & $50,0 \%$ & $77,3 \%$ \\
\hline & & 23 & $43,8 \%$ & $65,2 \%$ & $100 \%$ \\
\hline
\end{tabular}


Na correlação entre o acometimento linfonodal mesenterial e óbito observou-se diferença significante $(P<0,038)$ quando da comparação entre o grupo com e sem comprometimento linfonodal mesenterial. Todos os pacientes em que existia acometimento linfonodal mesenterial morreram por doença no seguimento realizado (Tabela 8).

TABELA 8 - Correlação entre o comprometimento linfonodal mesenterial e óbito pela doença

\begin{tabular}{cccccc}
\hline & & \multicolumn{4}{c}{ ÓBITO } \\
& & & Não & Sim & Total \\
\hline & \multirow{2}{*}{ Não } & No. casos & 21 & 27 & 48 \\
& & $43,8 \%$ & $56,3 \%$ & $100,0 \%$ \\
& & Total & $38,9 \%$ & $50,0 \%$ & $88,9 \%$ \\
\cline { 2 - 6 } COMPROMETIMENTO & & No casos & - & 6 & 6 \\
LINFONODAL & \multirow{2}{*}{ Sim } & $\%$ & - & $100,0 \%$ & $100,0 \%$ \\
MESENTERIAL & & Total & - & $11,1 \%$ & $11,1 \%$ \\
\cline { 2 - 6 } & \multirow{2}{*}{ Total } & No. casos & 21 & 33 & 54 \\
& & $\%$ & $38,9 \%$ & $61,1 \%$ & $100,0 \%$ \\
\hline
\end{tabular}

Dos 46 pacientes $(88,4 \%)$ que não apresentavam comprometimento linfonodal mesenterial, 25 faleceram (54,3\%), a média de sobrevivência foi de 143,5 meses e aos 60 meses a sobrevivência foi de $34,7 \%$. Com comprometimento linfonodal mesenterial houve 6 casos (11,5\%), 100\% dos pacientes faleceram, com média de sobrevivência de 32 meses e aos 60 meses $16,6 \%$ dos pacientes estavam vivos $(P<0,0032)$. Na análise da sobrevivência geral constatou-se que sobrevivência média de 102,5 meses e aos 60 meses houve sobrevivência de $31,8 \%$ dos pacientes.

\section{DISCUSSÃO}

Para que a neoplasia maligna assestada sobre o coto gástrico seja considerada uma nova entidade não relacionada à doença gástrica previamente tratada por intervenção cirúrgica, é necessário intervalo mínimo de tempo de 5 anos entre aquela intervenção e o diagnóstico.

Nesta observação, a idade dos pacientes com a doença (mediana de 64,5 anos) foi semelhante à observada em pacientes com estômago não operado (60-70 anos), o que pode indicar que a gastrectomia prévia não é um fator único de carcinogênese, fato semelhante observado por outros autores ${ }^{6,20,34}$.

É consenso que a reconstrução a Billroth II propicia maior refluxo do suco intestinal-bile-pancreático, o que favorece a carcinogênese por agressão à mucosa gástrica. A maioria dos pacientes estudados (cerca de 80\%) foi submetida a tal reconstrução na primeira operação e tiveram maior chance de invasão tumoral da linha de anastomose.

Estudos ${ }^{9,21}$ mostraram que a invasão linfonodal no câncer do coto gástrico é freqüente, porém de padrão não específico. Assim, utiliza-se a classificação habitual do câncer de estômago não operado para o seu estadiamento TNM, o que pode representar um problema quando da análise dos resultados encontrados devido à alteração anatômica que a operação prévia levou a acontecer.
Deste modo, a reconstrução do trânsito digestivo após a gastrectomia, mormente reconstrução a Billroth II e Y de Roux, introduz uma nova rota de disseminação linfonodal quando coloca os linfonodos, do mesentério e do intestino delgado, próximos à linha anastomótica que pode ou não estar invadida. Mesmo assim, não se encontram estudos na literatura médica que levem em conta esta alteração provocada pela gastrectomia e reconstrução do trato alimentar. Por outro lado como não se detectou distribuição particular do comprometimento linfonodal pelo adenocarcinoma de coto, torna-se compreensível a não existência de uma classificação à parte. A diferença de alteração anatômica imposta pelo cirurgião, quando da gastrectomia prévia, tornando a anatomia da região e mais especificamente da drenagem linfática muito variável entre os casos, é mais um fator que dificulta a criação de classificação única para o câncer de coto gástrico.

A invasão da linha anastomótica pelo tumor foi freqüente, o número de pacientes com linha invadida (75\%) e sem invasão (20,8\%) motivou o estudo destes dois grupos separadamente com o intuito de encontrar diferenças entre os resultados cirúrgicos obtidos, em especial quanto à sobrevivência tardia dos grupos diferentes de pacientes.

Houve invasão linfonodal regional e mesenterial com o acometimento tumoral da linha de anastomose, maior chance de óbito com esses eventos, porém menor probabilidade de recidiva tumoral quando havia invasão da linha de anastomose o dados estes concordantes com a literatura ${ }^{4,22}$. A fibrose da cicatriz cirúrgica da linha de anastomose pode interromper algumas vias de disseminação linfática para difusão da doença.

A invasão linfonodal também não seguiu padrão específico, mas foi freqüente, porém sem diferença estatística significante quanto a sua distribuição e número de linfonodos acometidos quando se comparou os grupos com e sem invasão linfonodal. É, portanto semelhante à distribuição quanto à ausência de acometimento linfonodal, acometimento de linfonodos perigástricos (N1) e acometimento de linfonodos da segunda estação (N2).

Muito embora a invasão linfonodal mesenterial seja muito prevalente no câncer de coto gástrico $(46 \%)^{31}$, no presente estudo tal fato não aconteceu.

Sabe-se que o sítio de maior prevalência de acometimento do câncer é próximo à linha de anastomose $e^{6,12,32}$, coincidindo com o resultado encontrado neste estudo (75\%).

A sobrevivência com a invasão tumoral da linha alcançou média de 112,51 meses e não foi diferente estatisticamente daqueles sem invasão: 89,99 meses. Tal observação não confirmou a hipótese inicial de que o acometimento da linha de anastomose favoreceria a disseminação através de metástases linfonodais do mesentério. Esta condição é considerada grave pois este acometimento é considerado metástase à distância (M1) e configurado estado avançado (IV). Todos os pacientes do presente estudo com tal acometimento linfonodal faleceram pela progressão da doença. Tal observação parece indicar que a metástase para os linfonodos mesentéricos se dá mais por razões intrínsecas 
à biologia do adenocarcinoma que pelas alterações da anatomia provocadas pela gastrectomia prévia.

Em estudo multifatorial em relação à recidiva tumoral, notou-se que no grupo de pacientes com comprometimento linfonodal teve maior porcentagem de recidiva tumoral $(62,5 \%)$ em relação ao grupo sem acometimento linfonodal $(37,5 \%)$ porém sem diferença significante entre os grupos $(P<0,201)$. Em relação à invasão tumoral mesenterial, o resultado foi semelhante, porém sem confiabilidade estatística, devido amostragem pequena. $\mathrm{Na}$ correlação entre invasão tumoral da linha de anastomose e recidiva tumoral mostrou-se que na presença de tumor na linha de anastomose, a recidiva é menor (20\%) quando comparada como grupo sem tumor na linha de anastomose $(62,5 \%)$ com diferença significante entre os grupos $(P<$ 0,023 ). No estudo de correlação entre recidiva tumoral e tipo histológico do tumor segundo a classificação de Nakamura não houve diferença significante para recidiva nos grupos de tipo intestinal $(70 \%)$ ou gástrico $(30 \%)$ e presença de recidiva tumoral, com $\mathrm{p}<0,603$. Percebeu-se que na correlação entre óbito e recidiva tumoral o número de óbitos foi maior em relação aos pacientes com recidiva, e a diferença foi significante entre o grupo sem recidiva tumoral $(P<0,003)$.

Dois achados significativos ocorreram no estudo estatístico de correlação entre idade e intervalo de tempo decorrido da operação de correção da doença benigna com a recidiva tumoral. Quanto menor a idade, maior a chance de recidiva tumoral $(P<0,020)$ e quanto menor o intervalo de tempo decorrido da operação de correção da doença benigna, maior a chance de recidiva tumoral $(P<0,089)$.

Os resultados aqui obtidos em especial o relativo bom prognóstico observado no tratamento cirúrgico aplicado (sobrevivência geral de 31,8\%) - associados à impossibilidade de identificar um padrão especial de comprometimento linfonodal, grande variabilidade anatômica após a gastrectomia prévia, não encontro de qualquer resultado que favoreça o conceito de que as aderências da intervenção cirúrgica prévia se constituiriam em nova via de disseminação neoplásica, não confirmação de que o acometimento da linha de anastomose representa obscurecimento dos resultados do tratamento aplicado -, sugerem que não há necessidade de se propor uma classificação especial para o câncer de coto gástrico, assim como um tipo de tratamento cirúrgico particularizado.

\section{CONCLUSÃO}

1. O câncer de coto gástrico não teve padrão de disseminação linfonodal específico. 2. A linha de anastomose sofre freqüente invasão tumoral. 3. Apesar de freqüente a invasão da linha anastomótica, ela por si só não apresenta correlação estatística significante com o comprometimento linfonodal regional ou mesenterial. 4. A presença de invasão linfonodal implica em sobrevida menor, em especial a invasão de linfonodos do mesentério. 5. A presença de acometimento neoplásico da linha anastomótica não se correlaciona com pior resultado de sobrevivência.

Carrasco ANGSN, Bresciani CJC, Perez RO, Zilbertein B, Cecconello I. The influence of tumor invasion in anastomotic line on survival of patient with gastric stump cancer. ABCD Arq Bras Cir Dig. 2008;21(2):55-60

ABSTRACT - Aim - To identify the lymph node metastatic pattern for gastric stump cancer; to quantify the anastomotic site invasion by tumor growth; to correlate the invasion of anastomotic site with metastasis in lymph node in general and mesenterial lymph node involvement and the survival. Methods - One hundred and thirteen patients with gastric stump cancer were retrospectively analyzed along with their medical records, surgical specimens and histopathologic exams. Results - seventy five percent of patients had tumoral invasion in the anastomotic site. In $66.7 \%$ there was invasion of the anastomotic site with metastatic lymph nodes. Nine percent had mesenterial lymph node invasion. Fatal cases occurred in $86,5 \%$ with metastatic lymph node, $64,7 \%$ with invasion of the anastomotic site and $100 \%$ with mesenterial lymph node invasion. Conclusions - Metastatic lymph node dissemination is not specific to gastric stump cancer and does not present a pattern in the lymph node dissemination. Although frequent the tumor invasion in anastomotic line, there is no significant statistical correlation with the invasion of regional or mesenterial lymph nodes. Lymph node invasion reduces survival, mainly when mesenterial lymph node dissemination is present. Tumor invasion in anastomotic line does not reduce survival.

HEADINGS - Stump gastric cancer. Surgical treatment. Gastric cancer

\section{REFERÊNCIAS}

1. Baulfour DC. Factors influencing the life expectancy of patients operated on for gastric ulcer. Ann Surg. 1922;76(3):405-8.

2. Bechi P, Amorosi A, Mazzanti R, Romagnoli P, Tonelli L. Gastric histology and fasting bile reflux after partial gastrectomy. Gastroenterology. 1987;93(2):33543.

3. Chen DN, Lee WJ, Lee PH, Chang KJ, Chen KM. Clinicopathologic characteristics and prognosis of gastric stump cancer. J Clin Gastroenterol. 1996;23(4):251-5.

4. Chen L, Tian H, Chen J, He ZG, Tao SF, Lokesh G, Peng SY. Surgical management of gastric stump cancer: a report of 37 cases. J Zhejiang Univ Sci B. 2005;6(1):38-42.

5. Correa P. Human gastric carcinogenesis: a multistep and multifactorial processFirst American Cancer Society Award Lecture on Cancer Epidemiology and Prevention. Cancer Res. 1992: 15;52(24):6735-40..
6. Domellof L, Eriksson S, Janunger K-G. Carcinoma and possible precancerous changes of the gastric stump after Billroth II resection. Gastroenterology. 1977;73(3):462-8.

7. Garrido Jr AB, Pinotti HW, Gama-Rodrigues J. Seqüelas das Ressecções Gástricas. In: Pinotti HW editor. Tratado de Clínica Cirúrgica do Aparelho Digestivo. São Paulo: Atheneu, 1994.vol.1. 600-18.

8. Giarelli L, Melato M, Stanta G, Bucconi S, Manconi R. Gastric resection: a cause of high frequency of gastric carcinoma. Cancer. 1983;52:1113-6.

9. Han SL, Hua YW, Wang CH, Ji SQ, Zhuang J. Metastatic pattern of lymph node and surgery for gastric stump cancer. Journal of Surgical Oncology. 2003;82(4):241-246.

10. Liavaag K. Cancer development in gastric stump after partial gastrectomy for peptic ulcer. Ann Surg. 1962;155:103-6. 
11. Kamata T. Experimental studies on the development of residual stomach cancer. Comparison of Billroth I and Billroth II. Nippon Geka Gakkai Zasshi. 1993;94(2):104-13.

12. Kobayashi S, Prolla JC, Kirsner JB. Late gastric carcinoma developing after surgery for benign conditions. Endoscopic and histologic studies of the anastomosis and diagnostic problems. Am J Dig Dis. 1970; 15(10):905-12.

13. Langhans P, Heger RA, Hohenstein J, Schlake W, Bünte H. Operation-sequel carcinoma of the stomach. Experimental studies of surgical techniques with or without resection. World J Surg. 1981;5(4):595-605.

14. Lipkin M. Biomarkers of increased susceptibility to gastrointestinal cancer: their development and application to studies of cancer prevention. Gastroenterology. 1987;92(4):1083-6.

15. Miwa K, Hattori T, Miyazaki I. Duodenogastric reflux and foregut carcinogenesis. Cancer. 1995;15:75(6):1426-32.

16. Moreaux J, Msika S. Carcinoma of the gastric cardia: surgical management and long-term survival. World J Surg. 1988;12(2):229-35.

17. Morgenstern L. Vagotomy, gastroenterostomy and experimental gastric cancer. Arch Surg. 1968;96(6):920-3.

18. Nakane Y, Okamura S, Boku T, Okusa T, Tanaka K, Hioki K. Prognostic differences of adenocarcinoma arising from the cardia and the upper third of the stomach. Am Surg. 1993;59(7):423-9.

19. Offerhaus GJ, Tersmette AC, Huibregtse K, van de Stadt J, Tersmette KW, Stijnen T, Hoedemaeker PJ, Vandenbroucke JP, Tytgat GN. Mortality caused by stomach cancer after remote partial gastrectomy for benign conditions: 40 years of follow up of an Amsterdam cohort of 2633 postgastrectomy patients. Gut. 1988;29(11):1588-90.

20. Ovaska JT, Havia TV, Kujari HP. Risk of gastric stump carcinoma after gastric resection for benign ulcer disease. Ann Chir Gynaecol. 1986;75(4):192-5

21. Parga GC, Saco AL, Iglesias RC, Grosso AH, Alvarado NC, González UM, Gil PG. Câncer de Estómago operado: estúdio descriptivo sobre la experiência de los últimos doce años. Revista Clínica Española. 1999;199(5):264-69.

22. Pointner R, Schwab G, Königsrainer A, Bodner E, Schmid KW. Early cancer of the gastric remnant. Gut. 1988 Mar;29(3):298-301.

23. Saegesser F, Jämes D. Cancer of gastric stump after partial gastrectomy (Billroth II principle) for ulcer. Cancer. 1972;29(5):1150-9.
24. Safatle-Ribeiro AV, Ribeiro Júnior U, Sakai P, Iriya K, Ishioka S, Gama-Rodrigues J. Gastric stump mucosa: is there a risk for carcinoma?. Arq Gastroenterol. 2001;38(4):227-31.

25. Stalberg H, Taksdal S. Stomach cancer following gastric surgery for benign conditions. Lancet. 1971 Nov27;2(7735):1175-7.

26. Stalnikowicz R, Benbassat J. Risk of gastric cancer after gastric surgery for benign disorders. Arch Intern Med. 1990 Oct;150(10):2022-6.

27. Schrumpf E, Serck-Hanssen A, Stadaas J, Myren J, Aune S, Osnes M. Mucosal changes in the gastric stump. 20-25 years after partial gastrectomy. Lancet. 1977 Sep3;2(8036):467-9.

28. Tanigawa N, Nomura E, Niki M, Shinohara H, Nishiguchi K, Okuzawa M, Toyoda M, Morita S. Clinical study to identify specific characteristics of cancer newly developed in the remnant stomach. Gastric Cancer. 2002; 5(1):23-8.

29. Tersmette AC, Offerhaus GJ, Tersmette KW, Giardiello FM, Moore GW, Tytgat GN, Vandenbroucke JP. Meta-analysis of the risk of gastric stump cancer: detection of high risk patient subsets for stomach cancer after remote partial gastrectomy for benign conditions. Cancer Res. 1990 15;50(20):6486-9.

30. Tofgaard C. Gastric cancer after peptic ulcer surgery. A historic prospective cohort investigation. Annals of Surgery. 1989;210(2):159-64.

31. Thorban S., Böttcher K., Etter M, Roder JD, Busch R, Siewert JR. Prognostic factors in gastric stump carcinoma. Ann Surg. 2000;231(2):188-94.

32. Tytgat GNJ, Offerhaus JGA, van de Stadt J, Huibregtse K. Early gastric stump cancer: macroscopic and microscopic appearance. Hepato Gastroenterol. 1989;36(2):103-108.

33. Vieira OM. Complicações Tardias (seqüelas) do tratamento Cirúrgico. In: Gama-Rodrigues JJ, Del Grande JC, Martinez JC. Tratado de Clínica Cirúrgica do Sistema Digestório. São Paulo: Atheneu, 2004.vol1.469-71.

34. Viste A, Bjornestad P, Opheim P, Skarstein A, Thunold J, Hartveit F, Eide GE, Eide TJ, Soreide O. Risk of carcinoma following gastric operations for benign disease: a historical cohort study of 3470 patients. Lancet. 1986 30:2(8505):502-5.

35. Yonemura Y, Ninomiya I, Tsugawa K, Masumoto H, Takamura H, Fushida S, Yamaguchi A, Miwa K, Miyazaki I. Lymph node metastases from carcinoma of the gastric stump. Hepato Gastroenterol. 1994;41(3):248-52.

Fonte de financiamento: não há Conflito de interesse: não há Recebido para publicação: 10/12/2007 Aceito para publicação: 18/02/2008 